\title{
Decision Problems for Linear Recurrence Sequences
}

\author{
Joel Ouaknine \\ Department of Computer Science \\ Oxford University, United Kingdom \\ Joel.Ouaknine@cs.ox.ac.uk
}

\begin{abstract}
.
Linear recurrence sequences (LRS), such as the Fibonacci numbers, permeate vast areas of mathematics and computer science. In this talk, we consider three natural decision problems for LRS, namely the Skolem Problem (does a given LRS have a zero?), the Positivity Problem (are all terms of a given LRS positive?), and the Ultimate Positivity Problem (are all but finitely many terms of a given LRS positive?). Such problems (and assorted variants) have applications in a wide array of scientific areas, such as theoretical biology (analysis of L-systems, population dynamics), economics (stability of supply-and-demand equilibria in cyclical markets, multiplier-accelerator models), software verification (termination of linear programs), probabilistic model checking (reachability and approximation in Markov chains, stochastic logics), quantum computing (threshold problems for quantum automata), discrete linear dynamical systems (reachability and invariance problems), as well as combinatorics, statistical physics, term rewriting, formal languages, cellular automata, generating functions, etc.

We shall see that these problems have deep and fascinating connections to rich mathematical concepts and conjectures, particularly in the fields of analytic and algebraic number theory, diophantine geometry and approximation, real algebraic geometry, mathematical logic, and complexity theory.
\end{abstract}

